\title{
Observer design for nonlinear systems with multi-rate sampled outputs - Application to attitude estimation
}

\author{
Tristan Bonargent ${ }^{* 1,2}$, Tomas Ménard ${ }^{1}$, Eric Pigeon ${ }^{1}$, Mathieu Pouliquen ${ }^{1}$ and Olivier Gehan ${ }^{1}$
}

\begin{abstract}
The present paper proposes an observer for a class of nonlinear systems with multi-rate sampled outputs. Each output can have irregular and aperiodic sampling rate independent of the others. This work extends previous studies on high gain observer to permit the estimation of multi-rated output systems. The stability is proved by using a Lyapunov approach, providing that the sampling rates of each outputs are respectively smaller than their associated upper bound. The observer is then applied to the attitude estimation problem.
\end{abstract}

Index Terms - High gain observer, multi-rate observers, nonlinear systems, sampled-data observers, attitude estimation

\section{INTRODUCTION}

The observer design for nonlinear systems has been studied for several decades. Various ideas have been proposed to address this problem. Among the many existing approaches, one can mention but a few, such as high gain observers [10], normal forms observers [4] or Lyapunov based observers [22], for example. Most of the existing observers in the literature consider only continuous-time measurements, while in practice, measurements are usually available only at some discrete instants. Furthermore, when many sensors are involved, several different sampling periods might have to be considered: one for each measured output. Indeed, nonlinear systems with multi-rate sampled outputs can be found in many disciplines. For instance, inertial measurement units use several sensors that may not have synchronous sampling rates [21], asynchronous measurements can be desired to reduce the energy use [19] or it can be also used for the fault detection of plants purposes [6]. Two main approaches have been explored when considering discrete time measurements: one can either discretize the system, in order to use a full discrete-time approach, or use an hybrid discrete-time measurement continuous-time system in order to keep the physical meaning of the continuous-time modeling of the system.

Following the first full discrete-time approach, Kalman filters are often used to deal with the considered problematic [11], [15], [25]. The estimation is made in two steps: the commonly called prediction and update stages. In [1], [2], due to the discrete nature of the system, sampled data of each output must be available at periodic instants. Two Kalman filters have been used in [8], one for regular sample and one for irregular samples. A multi-rate moving-horizon optimization-based observer has been considered in [18] to

\footnotetext{
${ }^{1}$ Normandie Univ, Unicaen, Ensicaen, LAC (EA 7478), 6 Bd Maréchal Juin, 14035 Caen Cedex, France

${ }^{2}$ KAD!, Espace Conquérant, 3 Place Jean Nouzille, 14000 Caen, France

*Corresponding author, e-mail: tristan.bonargent@unicaen.fr
}

reconstruct the state. An observer has been exposed in [20] under the assumption that the samples of the different outputs have a greatest common divisor. This efficient method can be hard to set up especially due to the important choice of the initialization. A sampler has been considered in [3], [16] in order to compute the outputs between two samples. These outputs estimates are then used to estimate the state. This permits to not have the same hold value between two samples.

The second approach estimates the state of the system in continuous-time from discrete-time measurements. This can be useful to implement a control law based on the continuous model or for monitoring purposes. A constant gain observer has been proposed in [17], using an exact linearization with the Lyapunovs auxiliary theorem [13]. More precisely, the authors use a continuous-time exact linearized differential equation to estimate the state and update the output when a data is sent by a sensor. The main drawback of this approach is that most systems cannot be easily linearized with the previous method due to the solving of partial differential equations, furthermore the design of the observer induces discontinuities of the estimated state that may not be desirable when used for control purposes such as in mechanical systems for instance. Another observer has been designed in [23] for multi-rate systems with delayed samples. The authors use a constant gain observer which avoid the linearization problem, but the considered sampling periods may be too small to be implemented in real conditions. A combination of a high-gain observer and an extended Kalman filter has been proposed in [9], which also estimate the state in continuous-time. It combines the two noise covariance matrices of a classic Kalman filter with some high-gain parameters. This allows the authors to reduce the noise impact while keeping the advantages of the highgain approach. Nevertheless, the estimated state is timediscontinuous, which might be problematic when used to feed a control law.

In this paper, we focus on a uniformly observable multioutput class of systems. We propose an observer which provides a continuous estimate of the state based on multirate outputs. The design considered here is based on the approach proposed in [7] for a class of uniformly observable systems and extended in [5] for perturbed systems and in [12] for a class of non-uniformly observable systems. A limitation of the existing results is that multi-rate outputs cannot be managed. An extension of these works is then presented in this paper in order to consider systems with several outputs 
each with different sampling.

The paper is organized as follows. In section II, the considered problem is stated. The proposed observer is described in section III with a proof of stability. Section IV contains some simulation results of a nonlinear multi-rate system in order to illustrate the behavior of the proposed approach. Finally section V concludes this paper.

\section{Problem statement}

In the present study, we consider a general class of nonlinear multi-output systems with multi-rate sampled data, which has been considered in [24] for the continuous outputs case with no dynamic uncertainties. The state space is separated into several blocks, each corresponding to an output. Each block dynamic is given by

$$
\left\{\begin{aligned}
\dot{x}_{1}^{i}(t) & =x_{2}^{i}(t)+\varphi_{1}^{i}(u(t), x(t)) \\
\dot{x}_{2}^{i}(t) & =x_{3}^{i}(t)+\varphi_{2}^{i}(u(t), x(t)) \\
\vdots & \\
\dot{x}_{\lambda_{i}-1}^{i}(t) & =x_{\lambda_{i}}^{i}(t)+\varphi_{\lambda_{i}-1}^{i}(u(t), x(t)) \\
\dot{x}_{\lambda_{i}}^{i}(t) & =\varphi_{\lambda_{i}}^{i}(u(t), x(t))+\varepsilon_{i}(t) \\
y^{i} & =x_{1}^{i}
\end{aligned}\right.
$$

for $i=1, \ldots, q$, with $x_{k}^{i} \in \mathbb{R}^{p_{i}}, k=1, \ldots, \lambda_{i}$, the block sub-states and $y^{i} \in \mathbb{R}^{p_{i}}$ the block output. One further denotes $n_{i}=\lambda_{i} p_{i}$ the dimension of the $i$-th block's state and $n=\sum_{i=1}^{q} n_{i}$ the dimension of the systems whole state. One assumes that the nonlinear functions $\varphi_{k}^{i}$ possess a particular triangular structure according to each block and between blocks, that is:

$$
\begin{aligned}
& \text { - } \varphi_{k}^{i}(u, x)=\varphi_{p}^{i}\left(u, x^{1}, x^{2}, \ldots, x^{i-1}, x_{1}^{i}, x_{2}^{i}, \ldots, x_{k}^{i}\right) \\
& \text { for } 1 \leq k \leq \lambda_{i}-1 \\
& \text { - } \varphi_{\lambda_{i}}^{i}(u, x)=\varphi_{\lambda_{i}}^{i}\left(u, x^{1}, x^{2}, \ldots, x^{i}\right)
\end{aligned}
$$

The functions $\varepsilon_{i}: \mathbb{R}^{+} \rightarrow \mathbb{R}^{p_{i}}$ represent the dynamic uncertainties and can depend on the state or on unknown parameters.

The outputs are assumed to be available only at some sampling instants, that is

$$
y^{i}\left(t_{k}^{i}\right)=x_{1}^{i}\left(t_{k}^{i}\right)
$$

where the $t_{k}^{i}$ represent the sampling instants of the output of the $i$-th block's output and verify

$$
0 \leq t_{0}^{i}<t_{1}^{0}<\cdots<t_{k}^{i}<t_{k+1}^{i}<\ldots
$$

The time between two successive measurements of the $i$-th block, denoted $\tau_{k}^{i}=t_{k+1}^{i}-t_{k}^{i}$, can vary and can be different for each block. In fact, each output can have irregular and aperiodic sampling rates independent of the other outputs. One further denotes $\tau_{M}^{i}=\max _{k} \tau_{k}^{i}$ the upper bound on the set of sampling periods of the $i$-th block.

In the following, the dynamics of each block, $i=1, \ldots, q$ given by (1) will be written in an equivalent way in the following compact form:

$$
\left\{\begin{aligned}
\dot{x}^{i}(t) & =A_{i} x^{i}(t)+\varphi^{i}(u(t), x(t))+B_{i} \varepsilon_{i}(t) \\
y^{i}\left(t_{k}^{i}\right) & =C_{i} x^{i}\left(t_{k}^{i}\right)
\end{aligned}\right.
$$

with $x^{i}=\left[\begin{array}{llll}\left(x_{1}^{i}\right)^{T} & \left(x_{2}^{i}\right)^{T} & \ldots & \left(x_{\lambda_{i}}^{i}\right)^{T}\end{array}\right]^{T} \in \mathbb{R}^{n_{i}}$, $\varphi^{i}(u, x)=\left[\begin{array}{lll}\left(\varphi_{1}^{i}(u, x)\right)^{T} & \left(\varphi_{2}^{i}(u, x)\right)^{T} \ldots\left(\varphi_{\lambda_{i}}^{i}(u, x)\right)^{T}\end{array}\right]^{T}$ and $A_{i}, B_{i}, C_{i}$ are the corresponding matrices, that is

$$
A_{i}=\left[\begin{array}{cccc}
0 & I_{p_{i}} & & 0 \\
\vdots & & \ddots & \\
0 & \ldots & 0 & I_{p_{i}} \\
0 & \ldots & 0 & 0
\end{array}\right] B_{i}=\left[\begin{array}{llll}
0 & \ldots & 0 & I_{p_{i}}
\end{array}\right]^{T}=\left[\begin{array}{llll}
I_{p_{i}} & 0 & \ldots & 0
\end{array}\right]
$$

The aim of the present study is to design an observer that provides continuous-time estimate of the state of the MIMO system (4) from the multi-rated output samples (2). The conditions of convergence concerning the upper bounds of the sampling rates as well as the observer structure will be exposed in the next section.

\section{A. Observer's design}

\section{MAIN RESUlTS}

The proposed observer is given by

$$
\begin{aligned}
& \dot{\hat{x}}^{i}(t)=A_{i} \hat{x}^{i}(t)+\varphi^{i}(u, \hat{x}(t)) \\
& \quad-\theta^{\delta_{i}} \Delta_{i}^{-1}(\theta) \Gamma^{i} e^{-\theta^{\delta_{i}} \Gamma_{1}^{i}\left(t-t_{k}^{i}\right)}\left(C_{i} \hat{x}^{i}\left(t_{k}^{i}\right)-y^{i}\left(t_{k}^{i}\right)\right)
\end{aligned}
$$

for $t \in\left[t_{k}^{i}, t_{k+1}^{i}\right), i=1, \ldots, q$, where $\hat{x}^{i} \in \mathbb{R}^{n_{i}}$ is the estimate of $x^{i}$. The diagonal matrix $\Delta_{i}(\theta)$ is defined as

$$
\Delta_{i}(\theta)=\operatorname{diag}\left(I_{p_{i}}, \frac{1}{\theta^{\delta_{i}}} I_{p_{i}}, \ldots, \frac{1}{\theta^{\delta_{i}\left(\lambda_{i}-1\right)}} I_{p_{i}}\right)
$$

with $\theta>1$ a tuning parameter of the high gain observer. The powers $\delta_{i}$ of $\theta$ in the matrix $\Delta_{i}(\theta)$ are defined as

$$
\delta_{i}=\lambda_{i+1} \delta_{i+1}, \text { for } i=1, \ldots, q-1 \text { and } \delta_{q}=1
$$

The gains $\Gamma^{i} \in \mathbb{R}^{n_{i}}$ are chosen such that $\bar{A}_{i} \triangleq A_{i}-\Gamma^{i} C_{i}$ is Hurwitz. It should be noted that, given the particular block structure of $A_{i}$ and $C_{i}, \Gamma_{i}$ can be chosen in the following form

$$
\Gamma^{i}=\left[\begin{array}{llll}
\Gamma_{1}^{i} I_{p_{i}} & \Gamma_{2}^{i} I_{p_{i}} & \ldots & \Gamma_{\lambda_{i}}^{i} I_{p_{i}}
\end{array}\right]
$$

with $\Gamma_{1}^{i}, \ldots, \Gamma_{\lambda_{i}}^{i} \in \mathbb{R}$.

Since the matrices $\bar{A}_{i}$ are Hurwitz, there exist positivedefinite matrices $P^{i}$ and positive reals $\mu^{i}$ such that

$$
P^{i} \bar{A}_{i}+\bar{A}_{i}^{T} P^{i} \leq-2 \mu^{i} I_{\lambda_{i}}, \quad i=1, \ldots, q .
$$

The maximal and minimal eigenvalues of $P^{i}$ are denoted respectively $\lambda_{M}^{i}$ and $\lambda_{m}^{i}$ and $\lambda_{M}=\max _{i} \lambda_{M}^{i}, \lambda_{m}=$ $\min _{i} \lambda_{m}^{i}, \sigma=\sqrt{\frac{\lambda_{M}}{\lambda_{m}}}, \mu=\min _{i} \mu^{i}$.

Remark 1: The class of systems considered in this paper is the same as the one presented in [24], but the powers $\delta_{i}$ of $\theta$ don't have the same definition. This is done to limit the effects of the uncertainties on the performance of the proposed observer.

\section{B. Convergence analysis}

Some assumptions must be first considered in order to obtain a correct estimation of the continuous state.

A1 The whole state $x=\left[\left(x^{1}\right)^{T}\left(x^{2}\right)^{T} \ldots\left(x^{q}\right)^{T}\right]^{T}$ is bounded, that is, $x(t) \in \mathbb{X}, \forall t \geq 0$, where $\mathbb{X}$ is a compact subset of $\mathbb{R}^{n}$.

A2 The functions $\varphi^{i}(u, x)$ are $L^{i}$ Lipschitz with respect to $x$ uniformly in $u$, i.e. there exists $L^{i}>0$ such that for $u \in \mathbb{U}$ and $x, \bar{x} \in \mathbb{R}^{n},\left\|\varphi^{i}(u, x)-\varphi^{i}(u, \bar{x})\right\| \leq$ $L^{i}\|x-\bar{x}\|$. One further denotes $L=\max _{i} L^{i}$. 
A3 The functions $\varepsilon_{i}$ are bounded, that is, there exists $\delta_{\varepsilon}^{i}>0$ such that $\left\|\varepsilon_{i}(t)\right\| \leq \delta_{\varepsilon}^{i}$ for all $t \geq 0$.

A4 The input $u$ is bounded, that is $u(t) \in \mathbb{U}, \forall t \geq 0$ where $\mathbb{U}$ is a compact subset of $\mathbb{R}^{s}$.

One can now states the convergence result.

Theorem 1: Consider system (4) subject to assumptions A1, A2, A3 and A4. Assume that $\theta \geq 1$ satisfies

$$
\frac{\theta^{\delta_{i}}}{2} \geq L^{i} \frac{\sqrt{n^{i}}}{\mu^{i}} \quad \text { for } \quad i=1, \ldots, q
$$

with $L^{i}$ the Lipschitz constant of $\varphi^{i}, n_{i}$ the size of $i$-th block state $x^{i}$ and $\mu^{i}$ defined by (9). Then, if, for each block $i=1, \ldots, q$, the upper bound on the sampling periods $\tau_{M}^{i}$ is chosen such that

$$
\tau_{M}^{i}<\tau_{0}^{i} \text { for } i \in[1, \ldots, q]
$$

with $\tau_{0}^{i}=\min \left(\frac{(\sqrt{2}-1) \sqrt{\lambda_{m}^{i}}}{4 \sqrt{\lambda_{M}^{i}}\left\|\Gamma^{i}\right\|\left(\theta^{\delta_{i}}+L^{i}\right)}, \frac{\sqrt{2 \lambda_{m}^{i}}}{\mu^{i}} \theta^{-\delta_{i}}\right), \lambda_{M}^{i}$ is the maximum eigenvalue of $P^{i}$ and $\lambda_{m}$ is defined in III-A, then there exists $\bar{\alpha}_{i} \geq 0$ and $\bar{\beta}_{i} \geq 0$, with $\bar{\beta}_{i}$ independent of $\theta$ and the uncertainties, such that the following inequality holds true for $i=1, \ldots, q$

$$
\left\|x^{i}(t)-\hat{x}^{i}(t)\right\| \leq \bar{\alpha}_{i} e^{-\frac{1}{4} \theta^{\delta_{i}} t}+\bar{\beta}_{i} \theta^{-\delta_{i}} \sum_{k=1}^{i} \delta_{\varepsilon}^{k}
$$

Remark 2: Theorem 1 shows the exponential convergence of the errors $\left\|x^{i}(t)-\hat{x}^{i}(t)\right\|$ toward a ball centered at the origin, whose radius is proportional to $\theta^{-\delta_{i}} \sum_{k=1}^{i} \delta_{\varepsilon}^{k}$. In other words, the effect of the uncertainties can be lessened by increasing the parameter $\theta$, as long as the inequality (11) is respected.

Remark 3: Equation (11) represents the condition of convergence with regard to the sampling rates. One can note that if $\theta$ increases, then $\tau_{M}^{i}$ decreases. Thus, the faster the observer, the smaller the sampling periods considered can be.

Before starting with the proof of theorem 1, the following result must be stated.

Lemma 1: Let $w:[-\tau,+\infty) \rightarrow \mathbb{R}$ be a $C^{1}$ function verifying the following inequality

$$
\frac{d}{d t}\left(w^{2}(t)\right) \leq-a w^{2}(t)+b \int_{t-\tau}^{t} w^{2}(s) d s+c e^{-d t}+k
$$

with $a>0, b \geq 0, \tau>0, c \geq 0, d>0, k \geq 0$ and $\tau<$ $\min \left(\frac{\sqrt{2}-1}{2} \frac{a}{b}, \frac{1}{\sqrt{2} a}\right)$. Then there exists $\bar{\alpha} \geq 0$ such that the following inequality holds true

where $\bar{\beta}=\min \left(\frac{a}{2}, d\right)$.

$$
w(t)^{2} \leq \bar{\alpha} e^{-\bar{\beta} t}+\frac{2 k}{a}
$$

Sketch of proof 1 (of Lemma 1): Let us denote $\alpha=\frac{a}{\sqrt{2}}$, $\beta=\frac{b}{a}\left(\frac{e^{\alpha \tau}-1}{\alpha}\right)$ and $\gamma=1-\beta$. Then, one can prove that $\beta \in\left(0,1-\frac{1}{\sqrt{2}}\right)$, which directly implies that $\gamma \in\left(\frac{1}{\sqrt{2}}, 1\right)$. Let us now define the following candidate Lyapunov function

$$
\Theta\left(w_{t}\right)=w^{2}(t)+b \int_{0}^{\tau} \int_{t-s}^{t} e^{\gamma \alpha(\nu-t+s)} w^{2}(\nu) d \nu d s
$$

where $w_{t}(s)=w(t+s), s \in[-\tau, 0]$. Then, its derivative can be over-valued in the following way

$$
\dot{\Theta}\left(w_{t}\right) \leq-\frac{a}{2} \Theta\left(w_{t}\right)+c e^{-d t}+k
$$

Finally applying the comparison lemma [14, lemma 3.4 p102] concludes the proof.

Proof 1 (of Theorem 1):

The proof is divided into three main parts. In part 1), the derivatives of the errors between the state and the estimated state are exposed together with a suitable change of coordinates. Then, some candidate Lyapunov functions are considered and an over-valuation of their derivatives are derived in part 2). Finally, an induction reasoning is used, in part 3), to conclude.

1) Error dynamics:

Let $t \in \mathbb{R}$ and define $k_{i} \in \mathbb{N}$ as

$$
k_{i}=\max \left\{k \in \mathbb{N} \mid t_{k}^{i}<t\right\}, \quad i=1, \ldots, q .
$$

The observation error dynamics of the $i$-th block, defined by $\tilde{x}^{i}(t)=\hat{x}^{i}(t)-x^{i}(t)$, can be written as follows.

$$
\begin{aligned}
& \dot{\tilde{x}}^{i}(t)=A_{i} \tilde{x}^{i}(t)+\Phi^{i}(u(t), \hat{x}(t), x(t)) \\
& \quad-\theta^{\delta_{i}} \Delta_{i}^{-1}(\theta) \Gamma^{i} e^{-\theta^{\delta_{i}} \Gamma_{1}^{i}\left(t-t_{k_{i}}^{i}\right)} C_{i} \tilde{x}^{i}\left(t_{k_{i}}^{i}\right)-B_{i} \varepsilon^{i}(t)
\end{aligned}
$$

where $\Phi^{i}(u(t), \hat{x}(t), x(t))=\varphi^{i}(u(t), \hat{x}(t))-\varphi^{i}(u(t), x(t))$. One denotes now $\bar{x}^{i}=\theta^{-\sigma_{i}} \Delta_{i}(\theta) \tilde{x}^{i}$, with $\sigma_{i}=\lambda_{1} \delta_{1}-$ $\lambda_{i} \delta_{i}$ then, using the equalities $\Delta_{i}(\theta) A_{i} \Delta_{i}^{-1}(\theta)=\theta^{\delta_{i}} A_{i}$ and $C_{i} \Delta_{i}^{-1}(\theta)=C_{i}$ yields

$$
\begin{array}{r}
\dot{\bar{x}}^{i}(t)=\theta^{\delta_{i}} \bar{A}_{i} \bar{x}^{i}(t)+\theta^{-\sigma_{i}} \Delta_{i}(\theta) \Phi^{i}(u(t), \hat{x}(t), x(t)) \\
+\theta^{\delta_{i}} \Gamma^{i} z^{i}(t)-\theta^{-\sigma_{i}} \Delta_{i}(\theta) B_{i} \varepsilon^{i}(t)
\end{array}
$$

with $z^{i}(t)=C^{i} \bar{x}^{i}(t)-e^{-\theta^{\delta_{i}} \Gamma_{1}^{i}\left(t-t_{k_{i}}^{i}\right)} C^{i} \bar{x}^{i}\left(t_{k_{i}}^{i}\right)$.

2) Lyapunov function:

Consider the candidate Lyapunov functions $V^{i}\left(\bar{x}^{i}\right)=$ $\left(\bar{x}^{i}\right)^{T} P^{i} \bar{x}^{i}$. For $t \in\left[t_{k_{i}}^{i}, t_{k_{i}+1}^{i}\left[\right.\right.$, the derivative of $V^{i}\left(\bar{x}^{i}(t)\right)$ can be over-valued as follows

$\dot{V}^{i}\left(\bar{x}^{i}\right) \leq-2 \theta^{\delta_{i}} \mu^{i}\left\|\bar{x}^{i}\right\|^{2}+2\left\|P^{i} \bar{x}^{i}\right\|\left\|\theta^{-\sigma_{i}} \Delta_{i}(\theta) \Phi^{i}(u, \hat{x}, x)\right\|$ $+2 \theta^{\delta_{i}}\left\|P^{i} \bar{x}^{i}\right\|\left\|\Gamma^{i}\right\|\left\|z^{i}\right\|+2 \theta^{-\sigma_{i}}\left\|P^{i} \bar{x}^{i}\right\|\left\|\Delta_{i}(\theta) B_{i} \varepsilon^{i}\right\|$

Since $\left\|P^{i} \bar{x}^{i}\right\| \leq \sqrt{\lambda_{M}^{i}} \sqrt{V^{i}\left(\bar{x}^{i}\right)}$, one obtains

$$
\begin{aligned}
\dot{V}^{i}\left(\bar{x}^{i}\right) & \leq-2 \theta^{\delta_{i}} \frac{\mu^{i}}{\lambda_{m}^{i}} V^{i}\left(\bar{x}^{i}\right) \\
& +2 \sqrt{\lambda_{M}^{i}} \sqrt{V^{i}\left(\bar{x}^{i}\right)}\left\|\theta^{-\sigma_{i}} \Delta_{i}(\theta) \Phi^{i}(u, \hat{x}, x)\right\| \\
& +2 \theta^{\delta_{i}} \sqrt{\lambda_{M}^{i}} \sqrt{V^{i}\left(\bar{x}^{i}\right)}\left\|\Gamma^{i}\right\|\left\|z^{i}(t)\right\| \\
& +2 \theta^{-\sigma_{i}-\delta_{i}\left(\lambda_{i}-1\right)} \sqrt{V^{i}\left(\bar{x}^{i}\right)} \sqrt{\lambda_{M}^{i}}\left\|\varepsilon^{i}(t)\right\|
\end{aligned}
$$

One now gets an over-valuation of $\left\|\theta^{-\sigma_{i}} \Delta_{i}(\theta) \Phi^{i}\right\|$ and $\left\|z^{i}(t)\right\|$ :

On one hand, one has

$$
\begin{aligned}
\left\|z^{i}(t)\right\| \leq \frac{\theta^{\delta_{i}}+L^{i}}{\sqrt{\lambda_{m}^{i}}} \int_{t-\tau_{M}^{i}}^{t} \sqrt{V^{i}\left(\bar{x}^{i}(s)\right)} d s \\
+\frac{L^{i}}{\sqrt{\lambda_{m}}} \int_{t-\tau_{M}^{i}}^{t} \sum_{k=1}^{i-1} \sqrt{V^{k}\left(\bar{x}^{k}(s)\right)} d s
\end{aligned}
$$

On the other hand, using classical high-gain techniques with $\theta \geq 1$ leads to

$$
\left\|\theta^{-\sigma_{i}} \Delta_{i}(\theta) \Phi^{i}(u, \hat{x}, x)\right\|
$$




$$
\leq \frac{\sqrt{n_{i}} L^{i}}{\sqrt{\lambda_{m}}}\left(\sum_{k=1}^{i-1} \sqrt{V^{k}\left(\bar{x}^{k}\right)}\right)+\frac{\sqrt{n_{i}} L^{i}}{\sqrt{\lambda_{m}^{i}}} \sqrt{V^{i}\left(\bar{x}^{i}\right)}
$$

Thus, by using inequalities (21), (22) and (23), denoting $C_{0}^{i}=\frac{\mu^{i}}{\sqrt{\lambda_{m}^{i}}}, C_{1}^{i}=\frac{\sqrt{n_{i}}}{\sqrt{\lambda_{m}}}, C_{2}^{i}=\frac{\sqrt{n_{i}}}{\sqrt{\lambda_{m}^{i}}}, C_{3}^{i}=\frac{\sqrt{\lambda_{M}^{i}}\left\|\Gamma^{i}\right\|}{\sqrt{\lambda_{m}^{i}}}$ and $C_{4}^{i}=\frac{\sqrt{\lambda_{M}^{i}}\left\|\Gamma^{i}\right\|}{\sqrt{\lambda_{m}}}$ and choosing $\theta$ verifying $-\theta^{\delta_{i}} C_{0}^{i}+L^{i} C_{2}^{i} \leq$ $-C_{0}^{i} \frac{\theta^{\delta_{i}}}{2}$, one obtains

$$
\begin{aligned}
\frac{d}{d t}\left(\sqrt{V^{i}}\right) & \leq-C_{0}^{i} \frac{\theta^{\delta_{i}}}{2} \sqrt{V^{i}\left(\bar{x}^{i}\right)}+L^{i} C_{1}^{i}\left(\sum_{k=1}^{i-1} \sqrt{V^{k}\left(\bar{x}^{k}\right)}\right) \\
& +C_{4}^{i} \theta^{\delta_{i}} L^{i} \int_{t-\tau_{M}^{i}}^{t} \sum_{k=1}^{i-1} \sqrt{V^{k}\left(\bar{x}^{k}(s)\right)} d s \\
& +C_{3}^{i} \theta^{\delta_{i}}\left(\theta^{\delta_{i}}+L^{i}\right) \int_{t-\tau_{M}^{i}}^{t} \sqrt{V^{i}\left(\bar{x}^{i}(s)\right)} d s \\
& +\theta^{-\sigma_{i}-\delta_{i}\left(\lambda_{i}-1\right)} \sqrt{\lambda_{M}^{i}} \delta_{\varepsilon}^{i}
\end{aligned}
$$

3) Induction reasoning:

One proceeds by induction to prove that there exist $\bar{\alpha}_{i} \geq 0$ and $\bar{\beta}_{i} \geq 0$ such that

$$
\sqrt{V^{i}\left(\bar{x}^{i}(t)\right)} \leq \bar{\alpha}_{i} e^{-\frac{1}{4} \theta^{\delta_{i}} t}+\theta^{-\delta_{1} \lambda_{1}} \bar{\beta}_{i} \sum_{k=1}^{i} \delta_{\varepsilon}^{i}
$$

for $i=1, \ldots, q$, where $\bar{\beta}_{i}$ is independent of $\theta$, and provided that each upper bound on the sampling period $\tau_{M}^{i}$ verifies $\tau_{M}^{i}<\tau_{0}^{i}$ with $\tau_{0}^{i}=\min \left(\frac{(\sqrt{2}-1) C_{0}^{i}}{4 C_{3}^{i}\left(\theta^{\delta_{i}}+L^{i}\right)}, \sqrt{2} \frac{\theta^{-\delta_{i}}}{C_{0}^{i}}\right)$.

Let us first consider the case $i=1$. One can directly apply the lemma 1 with $a=C_{0}^{1} \frac{\theta^{\delta_{1}}}{2}, b=C_{3}^{1} \theta^{\delta_{1}}\left(\theta^{\delta_{1}}+L^{1}\right), c=0$ and $k=\theta^{\delta_{i}-\delta_{1} \lambda_{1}} \sqrt{\lambda_{M}^{1}} \delta_{\varepsilon}^{1}$. Then there exists $\bar{\alpha}_{1} \geq 0$ such as

$$
\sqrt{V^{1}\left(\bar{x}^{1}(t)\right)} \leq \bar{\alpha}_{1} e^{-\frac{1}{4} \theta^{\delta_{1}} t}+\theta^{-\delta_{1} \lambda_{1}} \bar{\beta}_{1} \delta_{\varepsilon}^{1}
$$

with $\bar{\beta}_{1}=4 \sqrt{\lambda_{M}^{1}}$, provided that $\tau_{M}^{1}<\tau_{0}^{1}$.

Let us now consider the general case $i=2, \ldots, q$.

One assumes that there exist $\bar{\alpha}_{k} \geq 0$ and $\bar{\beta}_{k} \geq 0$ such as

$$
\sqrt{V^{k}\left(\bar{x}^{k}(t)\right)} \leq \bar{\alpha}_{k} e^{-\frac{1}{4} \theta^{\delta_{k}} t}+\theta^{-\delta_{1} \lambda_{1}} \bar{\beta}_{i-1} \sum_{j=1}^{k} \delta_{\varepsilon}^{j}
$$

provided that $\tau_{M}^{k}<\tau_{0}^{k}$, for $k=1, \ldots, i-1$.

The following over-valuations directly follows from the induction hypothesis and the fact that $\delta_{k}=\delta_{k+1} \lambda_{k+1}$ for $k=1, \ldots, q-1$

$$
\begin{gathered}
\sum_{j=1}^{i-1} \sqrt{V^{j}\left(\bar{x}^{j}(t)\right)} \leq \bar{\alpha} e^{-\frac{\theta^{\delta_{i-1}}}{4} t}+\bar{\beta} \theta^{-\delta_{1} \lambda_{1}} \sum_{k=1}^{i-1} \delta_{\varepsilon}^{k} \\
\int_{t-\tau_{M}^{i}}^{t} \sum_{j=1}^{i-1} \sqrt{V^{j}\left(\bar{x}^{j}(s)\right)} d s \leq \bar{\nu} e^{-\frac{\theta^{\delta_{i-1}}}{4} t}+\bar{\varsigma} \theta^{-\delta_{1} \lambda_{1}} \sum_{k=1}^{i-1} \delta_{\varepsilon}^{k}
\end{gathered}
$$

where $\bar{\alpha}=\sum_{k=1}^{i-1}\left(\bar{\alpha}_{k}\right), \quad \bar{\beta}=\sum_{k=1}^{i-1}\left(\bar{\beta}_{k}\right), \quad \bar{\nu}=$ $\bar{\alpha}\left(\frac{1-e^{-\frac{1}{4} \theta^{\delta_{i-1}} \tau_{M}^{i}}}{\frac{1}{4} \theta^{\delta_{i-1}}}\right)$ and $\bar{\varsigma}=\bar{\beta} \tau_{M}^{i}$.

Then, using inequality (24) with (28), one can apply lemma 1 with $a=C_{0}^{i} \frac{\theta^{\delta_{i}}}{2}, b=C_{3}^{i} \theta^{\delta_{i}}\left(\theta^{\delta_{i}}+L^{i}\right)$, $c=L^{i} C_{1}^{i} \bar{\alpha}+C_{4}^{i} \theta^{\delta_{i}} L^{i} \bar{\nu}, d=\frac{\theta^{\delta_{i-1}}}{4}$ and $k=$ $L^{i}\left(C_{1}^{i} \bar{\beta}+C_{4}^{i} \theta^{\delta_{i}} \bar{\zeta}\right) \theta^{-\delta_{1} \lambda_{1}}\left(\sum_{k=1}^{i-1} \delta_{\varepsilon}^{k}\right)+\theta^{\delta_{i}-\delta_{1} \lambda_{1}} \sqrt{\lambda_{M}^{i}} \delta_{\varepsilon}^{i}$.
Then there exists $\bar{\alpha}_{i} \geq 0$ such that

$$
\sqrt{V^{i}\left(\bar{x}^{i}(t)\right)} \leq \frac{\sqrt{\lambda_{m}^{i}}}{\theta^{\delta_{i} \lambda_{i}}} \bar{\alpha}_{i} e^{-\frac{1}{4} \theta^{\delta_{i}} t}+\frac{4 k}{\theta^{\delta_{i}}}
$$

since $\delta_{i-1} \geq \delta_{i}$ and provided that $\tau_{M}^{i}<\tau_{0}^{i}$.

Then, on gets

$\sqrt{V^{i}\left(\bar{x}_{i}(t)\right)} \leq \frac{\sqrt{\lambda_{m}^{i}}}{\theta^{\delta_{i} \lambda_{i}}} \bar{\alpha}_{i} e^{-\frac{1}{4} \theta^{\delta_{i}}}+\sqrt{\lambda_{m}^{i}} \bar{\beta}_{i} \theta^{-\delta_{1} \lambda_{1}} \sum_{k=1}^{i} \delta_{\varepsilon}^{i}$

with $\bar{\beta}_{i}=\frac{1}{\sqrt{\lambda_{m}^{i}}} \max \left\{\left(L^{i} C_{1}^{i} \bar{\beta}+C_{4}^{i} L^{i} \bar{\varsigma}\right), \sqrt{\lambda_{M}^{i}}\right\}$.

Finally, using the inequality $\left\|\tilde{x}^{i}\right\| \leq \frac{\theta^{\sigma_{i}+\delta_{i}\left(\lambda_{i}-1\right)}}{\sqrt{\lambda_{m}^{i}}} \sqrt{V^{i}\left(\bar{x}^{i}(t)\right)}$ and the equality $\sigma_{i}=\lambda_{1} \delta_{1}-\lambda_{i} \delta_{i}$ yields

$$
\left\|\tilde{x}^{i}(t)\right\| \leq \bar{\alpha}_{i} e^{-\frac{1}{4} \theta^{\delta_{i}} t}+\bar{\beta}_{i} \theta^{-\delta_{i}} \sum_{k=1}^{i} \delta_{\varepsilon}^{k}
$$

which concludes the proof.

\section{ATtitude Estimation}

\section{A. Presentation of the model}

The proposed observer is applied through simulations to the attitude estimation problem. The aim is to estimate the attitude of a rigid body using the measurements commonly found in inertial measurement units i.e. acceleration, magnetic field and angular velocity.

The desired attitude will be represented by the time varying matrix $R(t) \in S O(3)$. This matrix represents the rotation from an inertial reference frame to a body fixed frame. In the following, the geometric structure of $S O(3)$ will be omitted for the estimate, which means that the estimate of $R$ will belong to $\mathbb{R}^{3 \times 3}$ and not necessarily to $S O(3)$.

The acceleration and the magnetic field measurements are then given by

$$
a(t)=R^{T}(t) g_{0} \text { and } m(t)=R^{T}(t) m_{0}
$$

where $g_{0}=\left[\begin{array}{lll}0 & 0 & G\end{array}\right]^{T}$ is the standard gravity and $m_{0}=$ $\left[\begin{array}{lll}m_{a} & m_{b} & m_{c}\end{array}\right]^{T}$ the earth magnetic field. One further assumes that these two vectors are non-collinear. Thus, the cross product between $a$ and $m$, given by $c(t)=a(t) \times m(t)$, is non zero for all $t \geq 0$ and the vector observations $v(t)$, defined by

$$
v(t)=\left(\begin{array}{lll}
a^{T}(t) & m^{T}(t) & c^{T}(t)
\end{array}\right)^{T}
$$

is made up of three independent vectors.

The last measured signal is the angular velocity $\omega(t)$. It is given from a gyroscope which is assumed to be bias free. The dynamic of $R(t)$ is hence given by

$$
\dot{R}(t)=R(t)(\omega(t))_{\times}
$$

where $\omega_{\times} \in \mathbb{R}^{3 \times 3}$ is the skew-symmetric matrix associated to $\omega=\left(\begin{array}{lll}\omega_{1} & \omega_{2} & \omega_{3}\end{array}\right)^{T} \in \mathbb{R}^{3}$, which is defined by

$$
\omega_{\times}=\left(\begin{array}{ccc}
0 & -\omega_{3} & \omega_{2} \\
\omega_{3} & 0 & -\omega_{1} \\
-\omega_{2} & \omega_{1} & 0
\end{array}\right) .
$$

\section{B. Observer design}

In order to apply the observer presented in this paper to the attitude estimation problem, one first need to re-write system (33)-(34) into the form (1). The state of the system will be composed by the angular velocity and its first two 
derivatives on one part and by the vector observation $v$ on the other part. The dynamic of the first block, related to the angular velocity, is given by

$$
\left\{\begin{array}{l}
\dot{x}_{1}^{1}=x_{2}^{1} \\
\dot{x}_{2}^{1}=x_{3}^{1} \\
\dot{x}_{3}^{1}=\varepsilon \\
y^{1}=x_{1}^{1}=\omega
\end{array}\right.
$$

where $x_{1}^{1}, x_{2}^{1}, x_{3}^{1} \in \mathbb{R}^{3}$ represent, respectively, the angular velocity, its first and second derivatives, $\varepsilon \in \mathbb{R}^{3}$ represents the uncertainty on the dynamic and $y^{1}$ the measured output. The dynamic of the second block, related to the acceleration and magnetic measurement is given by

$$
\left\{\begin{array}{l}
\dot{x}_{1}^{2}=-S_{3}\left(x_{1}^{1}\right) x_{1}^{2} \\
y^{2}=x_{1}^{2}=v
\end{array}\right.
$$

where $S_{3}(\omega)=\operatorname{diag}\left(\omega_{\times}, \omega_{\times}, \omega_{\times}\right) \in \mathbb{R}^{9 \times 9}$.

It should be noted that the state of the second block is not $R(t)$, indeed, one first estimate here $v(t)$ in continuous time from the discrete time measurements, the rotation matrix is then estimated in a second step using the following relation

$$
R(t)=C_{2}^{-1} x_{1}^{2}(t)
$$

where $C_{2}$ is a constant and non singular matrix defined by

$$
C_{2}=\left[\begin{array}{ccc}
0 & 0 & G I_{3} \\
m_{a} I_{3} & m_{b} I_{3} & m_{c} I_{3} \\
-G m_{b} I_{3} & G m_{a} I_{3} & 0
\end{array}\right] .
$$

The proposed observer applied to this example can then be written as follows

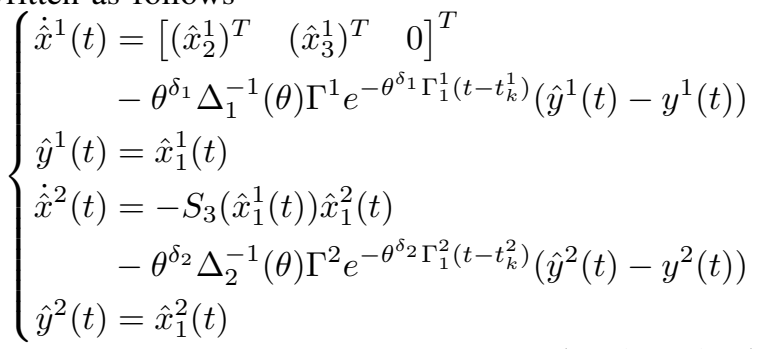

with $\delta_{1}=1, \delta_{2}=1, \Delta_{1}(\theta)=\operatorname{diag}\left(I_{3}, \frac{1}{\theta} I_{3}, \frac{1}{\theta^{2}} I_{3}\right)$ and $\Delta_{2}(\theta)=I_{9}$.

\section{Simulation results}

Two different simulations will be exposed in this section. For both simulations, the constant reference vectors are taken as $g_{0}=\left[\begin{array}{lll}0 & 0 & 9.81\end{array}\right]^{T}$ and $m_{0}=$ $\left[\begin{array}{lll}0.434 & -0.0091 & 0.908\end{array}\right]^{T}$. The gains $\Gamma^{1}$ and $\Gamma^{2}$ are chosen such that all the poles of $\left(A_{1}-\Gamma^{1} C_{1}\right)$ and $\left(A_{2}-\Gamma^{2} C_{2}\right)$ are located to -1 , that is

$$
\Gamma^{1}=\left(\begin{array}{lll}
3 I_{3} & 3 I_{3} & I_{3}
\end{array}\right)^{T} \quad \text { and } \quad \Gamma^{2}=I_{9} .
$$

The observer parameter $\theta$ has been fixed to 1.2. The evolution of the angular velocity is depicted on figure 1 . The sampling periods are chosen such that $\tau_{m}^{1} \leq \tau_{k}^{1} \leq \tau_{M}^{1}$ and $\tau_{m}^{2} \leq$ $\tau_{k}^{2} \leq \tau_{M}^{2}$, for all $k \in \mathbb{N}$, with $\tau_{m}^{1}=0.1 s, \tau_{M}^{1}=0.2 s$, $\tau_{m}^{2}=0.5 s$ and $\tau_{M}^{2}=0.75 s$. The sampling periods for both simulations are shown on figure 2. For the first simulation, the outputs are supposed to be available without noise. The errors between the reconstructed and real states are depicted on figure $3 \mathrm{a}$ ), b) and $\mathrm{c}$ ), the error $\|R(t)-\hat{R}(t)\|$ on

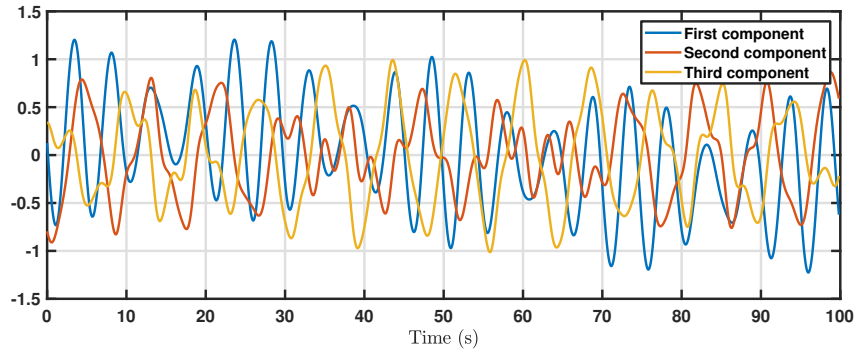

Fig. 1. Evolution of the angular velocity
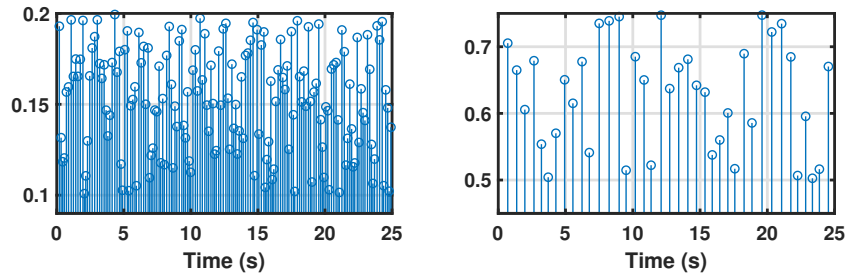

Fig. 2. Sampling periods of the outputs $y^{1}$ (left) and $y^{2}$ (right)

figure $3 \mathrm{~d}$ ) and the corresponding original and reconstructed Euler angles on figure $5 \mathrm{a}$ ), b) and c). As expected, the errors don't converge exactly to zero due to the uncertainties present in the first block, but it can be set as low as desired by increasing the parameter $\theta$. For the second simulation,

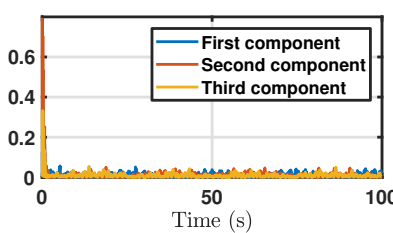

(a) Error between the components of $\omega$ and $\hat{\omega}$

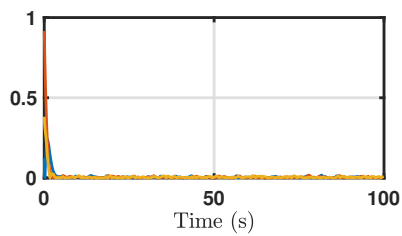

(c) Error between the component of $m$ and $\hat{m}$

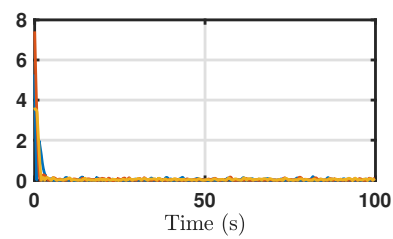

(b) Error between the components of $a$ and $\hat{a}$

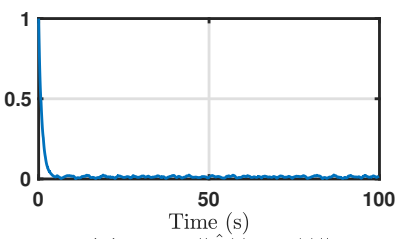

(d) Error $\|\hat{R}(t)-R(t)\|$
Fig. 3. Errors between the real and estimated outputs for the first simulation one assumes that the output $v(t)$, shown on figure 4 , is corrupted by a centered white noise. In fact the added white noises have respectively a variance of $\sigma_{a}=0.125 \mathrm{~m} / \mathrm{s}^{2}$ for the acceleration measurement and $\sigma_{m}=0.0625 \mu \mathrm{T}$ for the magnetic field measurement. The estimated Euler angles, depicted on figure $5 \mathrm{~d}$ ), e) and f) point out the good performances of the observer despite the noise. The errors between the real states and the estimated ones are exposed on figures 6. While the white noise present on the outputs induce higher bounds on the errors, compared to the first simulation, it should be noted that they are still satisfactory.

\section{CONCLUSION}

The problem of observer design for a large class of MIMO uniformly observable systems with multi-rate outputs has been considered in this paper. The proposed approach is based on an high-gain design which allow simple gain tuning.

The main feature of the observer is that each output can have independent and irregular sampling instants. Further- 


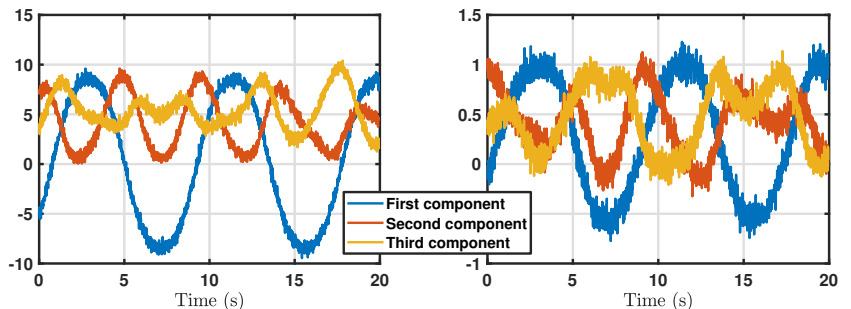

(a) Acceleration with the added noise

(b) Magnetic field with the added noise

Fig. 4. Acceleration and magnetic field with the added noise

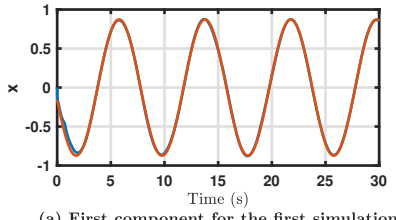

(a) First component for the first simulatio

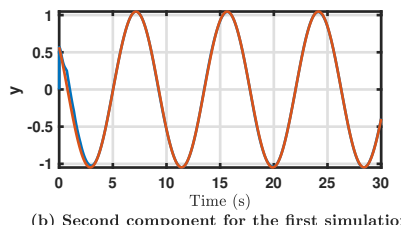

(b) Second component for the first simulation

(c) Third component for the first simulation

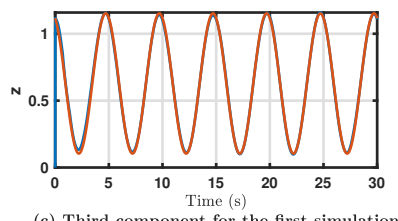

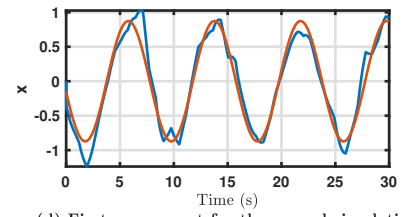

(d) First component for the second simulation

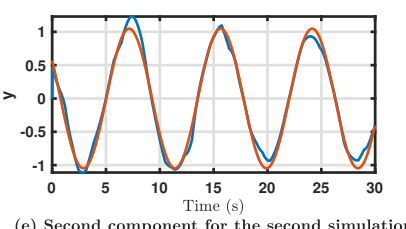

(e) Second component for the second simulation

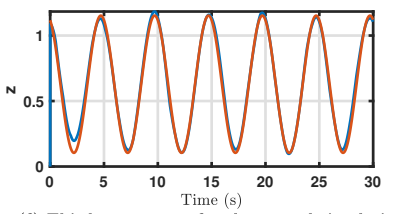

Fig. 5. Real and estimated Euler angles for both simulations

more, an analysis of the convergence is provided based on a Lyapunov approach and the effectiveness of the observer has been validated through simulations to the attitude estimation problem of a rigid body.

\section{REFERENCES}

[1] L. Armesto and J. Tornero. SLAM based on Kalman filter for multirate fusion of laser and encoder measurements. In 2004 IEEE/RSJ International Conference on Intelligent Robots and Systems (IROS) (IEEE Cat. No.04CH37566), volume 2, pages 1860-1865 vol.2, September 2004.

[2] L. Armesto, J. Tornero, and M. Vincze. Fast Ego-motion Estimation with Multi-rate Fusion of Inertial and Vision. The International Journal of Robotics Research, 26(6):577-589, June 2007.

[3] H. Beikzadeh and H. J. Marquez. Multirate Observers for Nonlinear Sampled-Data Systems Using Input-to-State Stability and DiscreteTime Approximation. IEEE Transactions on Automatic Control, 59(9):2469-2474, September 2014.

[4] D. Bestle and M. Zeitz. Canonical form observer design for non-linear time-variable systems. International Journal of Control, 38(2):419431, August 1983.

[5] I. Bouraoui, M. Farza, T. Ménard, R. Ben Abdennour, M. M'Saad, and H. Mosrati. Observer design for a class of uncertain nonlinear systems with sampled outputs - Application to the estimation of kinetic rates in bioreactors. Automatica, 55:78-87, 2015.

[6] M. S. Fadali. Observer-based robust fault detection of multirate linear system using a lift reformulation. Computers \& Electrical Engineering, 29(1):235-243, January 2003.

[7] M. Farza, M. M'Saad, M. L. Fall, E. Pigeon, O. Gehan, and R. Mosrati. Continuous-Discrete Time Observer for a class of MIMO Nonlinear Systems. IEEE Transactions on Automatic Control, pages 1060 1065, April 2014.

[8] A. Fatehi and B. Huang. Kalman filtering approach to multi-rate information fusion in the presence of irregular sampling rate and variable measurement delay. Journal of Process Control, 53:15-25, May 2017.

[9] A. Feddaoui, N. Boizot, E. Busvelle, and V. Hugel. High-gain extended

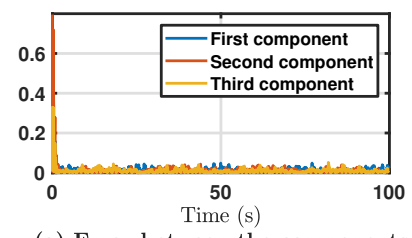
of $\omega$ and $\hat{\omega}$

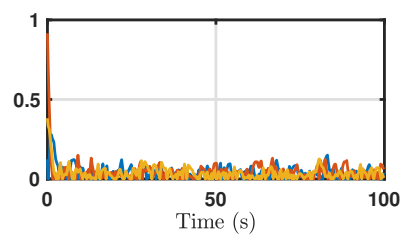

(c) Error between the components of $m$ and $\hat{m}$ (a) Error between the components

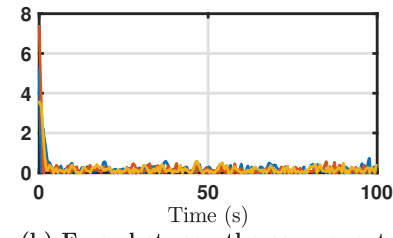
of $a$ and $\hat{a}$

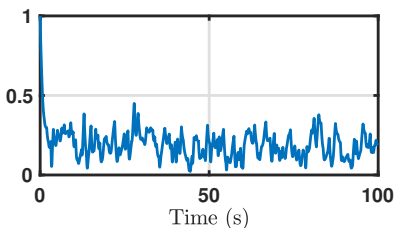

(d) Error $\|\hat{R}(t)-R(t)\|$ (b) Error between the components

Fig. 6. Errors between the real and estimated outputs for the second simulation

Kalman filter for continuous-discrete systems with asynchronous measurements. International Journal of Control, 0(0):1-14, October 2018.

[10] J. P. Gauthier, H. Hammouri, and S. Othman. A simple observer for nonlinear systems applications to bioreactors. IEEE Transactions on Automatic Control, 37(6):875-880, June 1992.

[11] A. Gopalakrishnan, N. S. Kaisare, and S. Narasimhan. Incorporating delayed and infrequent measurements in Extended Kalman Filter based nonlinear state estimation. Journal of Process Control, 21(1):119-129, January 2011.

[12] O. Hernández-González, M. Farza, T. Ménard, B. Targui, M. M’Saad, and C. M. Astorga-Zaragoza. A cascade observer for a class of MIMO non uniformly observable systems with delayed sampled outputs. Systems \& Control Letters, 98:86-96, December 2016.

[13] N. Kazantzis and C. Kravaris. Nonlinear observer design using Lyapunov's auxiliary theorem. Systems \& Control Letters, 34(5):241247, July 1998.

[14] Hassan K. Khalil. Nonlinear Systems. Prentice Hall, 2002.

[15] W. Li, S. L. Shah, and D. Xiao. Kalman filters in non-uniformly sampled multirate systems: For FDI and beyond. Automatica, 44(1):199208, January 2008.

[16] C. Ling and C. Kravaris. Multi-rate observer design for process monitoring using asynchronous inter-sample output predictions. AIChE Journal, 63(8):3384-3394, 2017.

[17] C. Ling and C. Kravaris. Multi-rate sampled-data observers based on a continuous-time design. In 2017 IEEE 56th Annual Conference on Decision and Control (CDC), pages 3664-3669, December 2017.

[18] R. López-Negrete and L. T. Biegler. A Moving Horizon Estimator for processes with multi-rate measurements: A Nonlinear Programming sensitivity approach. Journal of Process Control, 22(4):677-688, April 2012.

[19] A. Makni, H. Fourati, and A. Y. Kibangou. Adaptive Kalman filter for MEMS-IMU based attitude estimation under external acceleration and parsimonious use of gyroscopes. In 2014 European Control Conference (ECC), pages 1379-1384, June 2014.

[20] M. Moarref and L. Rodrigues. Observer design for linear multi-rate sampled-data systems. In 2014 American Control Conference, pages 5319-5324, June 2014.

[21] G. Nützi, S. Weiss, D. Scaramuzza, and R. Siegwart. Fusion of IMU and Vision for Absolute Scale Estimation in Monocular SLAM. Journal of Intelligent \& Robotic Systems, 61(1):287-299, January 2011.

[22] S. Raghavan and J. K. Hedrick. Observer design for a class of nonlinear systems. International Journal of Control, 59(2):515-528, February 1994.

[23] Y. Shen, D. Zhang, and X. Xia. Continuous observer design for a class of multi-output nonlinear systems with multi-rate sampled and delayed output measurements. Automatica, 75(Supplement C):127132, January 2017.

[24] H. Shim, Y. I. Son, and Jin H. Seo. Semi-global observer for multioutput nonlinear systems. Systems \& Control Letters, 42(3):233-244, March 2001.

[25] B. Sinopoli, L. Schenato, M. Franceschetti, K. Poolla, M. I. Jordan, and S. S. Sastry. Kalman filtering with intermittent observations. IEEE Transactions on Automatic Control, 49(9):1453-1464, September 2004. 